\title{
资源受限的深度学习: 挑战与实践
}

\author{
吴建金*，高斌斌，魏秀参，罗建豪 \\ 南京大学软件新技术国家重点实验室, 南京 210023 \\ *通信作者. E-mail: wujx2001@nju.edu.cn \\ 收稿日期: 2018-02-05; 接受日期: 2018-03-12; 网络出版日期: 2018-05-11 \\ 国家自然科学基金 (批准号: 61772256, 61422203) 资助项目
}

\begin{abstract}
摘要 深度学习近年来取得了突出进展, 然而, 深度学习模型需要占用大量的与计算相关的资源, 同 时其学习过程需要大量的数据与标记, 因此目前深度学习领域的一个热点是降低其对计算和数据资 源的渴求, 即研究资源受限的深度学习. 本文首先分析深度学习对资源的渴求及其导致的挑战, 然后 分别从数据、标记、计算资源受限 3 个方面对目前的研究进展简要描述, 并以我们在计算机视觉领 域的研究实践为例进行较详细的介绍.
\end{abstract}

关键词 深度学习, 资源受限, 数据资源, 标记资源, 计算资源

\section{1 引言}

深度学习这一名词可以被用来描述采用多层结构的机器学习模型, 以对大量复杂数据进行分析, 是一系列方法、模型、软件与硬件实现及其应用的统称. 近来, 深度学习在许多涉及大量、复杂数据 的应用方面取得了突破性进展, 主要包括图像及视频、音频、自然语言等. 例如, 在计算机视觉领域 经典的物体识别问题中, Krizhenvsky 等 ${ }^{[1]}$ 使用深度卷积神经网络模型 ${ }^{[2]}$ 对 120 万张物体图像进行 1000 个类别的分类, 其准确度远超计算机视觉领域近二十年来设计的其他方法; 在此之后, 新的模型 (如残差网络 ${ }^{[3]}$ 等) 将准确率继续大幅提高.

深度学习的成功受益于可用于训练模型的数据大幅提升 (如有标记的训练图像), 且可用的计算资 源显著增长 (特别是大量使用 GPU 用于并行计算). 然而, 经典的深度学习模型通常需要占用大量与 计算相关的资源, 如 CPU、GPU、内存、显存、硬盘、运行时间、电力等; 深度学习的另一个特点是其 学习过程需要大量的数据资源, 包括数据本身和对数据的标记 (如某张图像中包含的物体名称). 然而, 深度学习的应用场景通常需要实时且占用内存、计算等资源少的轻量级模型, 且大量用于训练的有标 记数据也难以取得. 因此, 目前深度学习领域的一个热点是研究新的方法与模型, 降低其对这些计算、 数据和标记资源的渴求, 即研究资源受限的深度学习.

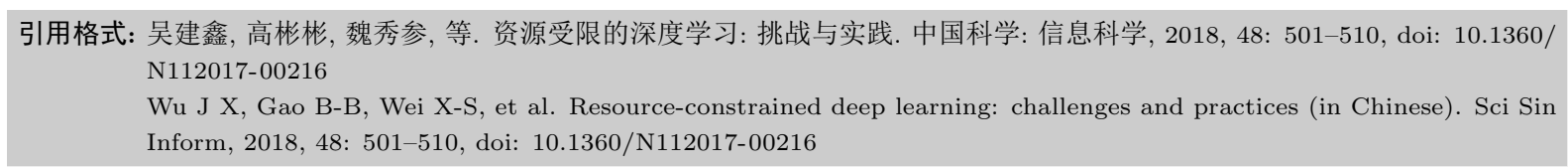


本文首先分析深度学习模型对各种计算、数据与标记资源的需求及其导致的困难与挑战, 然后分 别从数据、标记、计算资源受限这 3 个方面对目前的研究进展进行简要描述, 并以我们在计算机视觉 领域的研究实践为例进行较详细的介绍, 希望能引起对资源受限的深度学习这一方向更深入的思考.

\section{2 深度学习对资源的渴求及其面临的挑战}

机器学习方法通常分为训练 (或学习) 与测试 (或应用) 两个阶段. 在训练阶段, 使用多个训练样 本对某种机器学习模型进行训练; 训练完成后即可进行测试, 将模型应用到训练时未见过的新样本, 对 其进行预测. 很多经典的机器学习方法在训练阶段需要使用大量的资源, 在应用时则对资源需求较少. 但是, 深度学习无论是在训练还是应用时, 都需要大量的计算和/或数据资源.

深度学习对资源的渴求可以通过谷歌研究院最近的一篇论文来说明, 这篇论文 ${ }^{[4]}$ 使用超过 3 亿 张图像在 50 张 GPU 卡上使用异步并行计算方法训练, 在 2 个月的时间内仅能训练 4 轮, 但得到的卷 积神经网络模型准确度大大超过了以往的模型 (以往的模型使用约一百万张图像进行训练). 该文作 者据此得出结论: 即使图像标记的噪声很多, 使用更多训练图像和更大的模型有助于提高模型的准确 率. 然而, 这篇论文使用的数据与计算资源巨大, 类似的研究只能在极少数的研究机构才有条件进行, 不利于深度学习的研究与应用.

具体来说, 深度学习模型至少需要大量以下的各种资源:

数据. 机器学习需要大量训练样本以发现模型的最优参数, 而深度学习由于参数数量多、表达能 力强, 较其他方法需要更多的训练数据, 如文献 [4] 所示, 越多的训练样本通常意味着更高的准确率.

标记. 然而, 训练数据的获取需要消耗大量的资源. 在监督学习范式里, 数据需要有相应的标记 才可以进行学习; 而标记的获取则需要消耗大量的人力与财力. 例如, 在图像语义分割任务中, 标记一 张图像需要指出该图像中每一个像素的语义类别, 单张图像的标记需要半个小时才能完成; 在医学图 像分析等领域, 为图像提供标记 (如是否患有某种疾病) 只有医学专家才能完成.

计算. 深度学习, 无论是模型的学习 (训练) 还是模型的应用 (测试) 都需要大量的计算资源, 如 内存、显存、运算量等, 分别详述如下.

(1) 内存. 经典的深度学习模型有大量的参数, 以此获得强的表示能力. 例如, 常用的 VGG16 网 络 ${ }^{[5]}$ 有超过 $10^{8}$ 个参数, 占用超过 $500 \mathrm{M}$ 的内存空间, 这样的模型无法在手机等硬件资源受限的平 台上使用. 对这些模型进行训练需要占用更多的内存以保存学习过程中的中间结果 (如用来更新参数 的梯度、模型各处理层的激活图等).

(2) 显存. 当 GPU 被用于加速训练过程时, GPU 卡的显存大小也成为了另一个资源瓶颈. 在一些 复杂任务中, 例如将识别、分割、检测等任务融于单个网络的 Ubernet ${ }^{[6]}$, 即使是现在先进的 GPU 卡, 若不使用特殊算法, 其显存容量甚至都不足以处理单张输入图像, 给学习与测试都带来了极大的资源 压力.

(3) 运算量. 运算量体现在对 CPU 及 GPU 的占用率及占用时间上. 由于深度学习模型有大量参 数, 且需要进行大量的浮点运算, 其训练与测试阶段均要占据大量的 CPU 和/或 GPU 运算时间. 以训 练为例, 由于单个训练样本需要进行很多计算且有大量训练样本, 即使使用多个 GPU 卡加速, 大规模 深度模型的学习时间常以星期甚至月为单位 ${ }^{[4]}$. 而在测试、应用时, 尽管在有高性能 GPU 卡加速的 情况下可以实时完成很多任务, 在常见的资源受限平台上, 经典的深度学习模型不仅超出了平台能够 提供的内存、存储等资源, 其运行时间与 CPU 占用率也远远超标, 从而影响了深度学习的广泛应用. 
（4）其他. 此外, 其他资源如能源占用在深度学习的移动应用中也很重要. 参数众多、计算量大的 复杂模型无疑将消耗更多电力, 从而影响其在资源受限环境中的使用. 如果需要在这种环境中实时更 新模型 (即进行在线学习), 那么更需要降低深度学习对各种资源的需求.

如上所述, 在模型的训练学习和测试应用这两个阶段, 深度学习均面临着资源受限导致的各种挑 战. 如何在资源受限的环境中进行深度学习的训练与应用是目前学术界与工业界共同的研究热点之一, 下文将对这方面的工作进行简要的介绍, 并以我们的一些实践为例进行详细说明.

\section{3 减少深度学习对数据的依赖}

减少对大训练数据的依赖一直是深度学习的热点, 有两类方法具有代表性. 一类方法从已有的训 练样本产生虚拟样本, 从而增加训练数据的数量. 例如, 在图像识别领域, 很多模型在训练时将图像左 右翻转, 从而使得 $n$ 张训练图像扩充成了 $2 n$ 张. 随着生成式模型 (如生成式对抗模型 ${ }^{[7]}$ ) 变得逐渐可 用, 许多方法使用生成式模型来生成 (理论上可以无限多的) 虚拟训练样本, 并力图使虚拟样本具有与 真实训练样本相似的分布或性质 ${ }^{[8]}$.

另外一类方法则依赖预训练的模型. 例如, ImageNet ${ }^{1)}$ 是一个大型图像数据集, 它的子集 ILSVRC $2012^{[9]}$ 包含一百多万张训练图像, 很多方法使用其训练用于图像分类的深度网络模型, 如 VGG16 网 络. 由于该数据集包含了 1000 种不同物体的图像, 通常认为 VGG16 等网络包含了对于表示通用图像 有用的知识 (即通用图像表示): 当面对一个只有较少训练样本的新的学习任务时, 用这样的深度网络 的参数 (称为预训练的网络) 初始化一个新网络, 使用新问题中的少量训练数据对网络参数继续训练, 这个过程被称为微调 (fine-tuning). 若随机初始化新网络的参数并重新学习, 因为训练数据少, 网络的 错误率可能很高; 然而, 由于预训练的网络参数已经隐含了通用图像表示, 在此基础上进行微调, 即使 新问题的训练样本很少, 一般也能取得很好的效果. 除了物体识别之外, 微调策略被广泛应用于物体 检测 $[10]$ 、语义分割 ${ }^{[11]}$ 等任务中.

我们认为, 微调并不能完全、充分地利用预训练网络所包含的图像表示知识, 因此提出将预训练 网络用于无监督的细粒度图像检索任务 (即新问题中的训练图像不包含任何标记), 提出了 SCDA 方 法 ${ }^{[12]}$. 事实上, SCDA 方法仅仅利用了预训练模型中包含的知识, 并不需要训练数据, 故而能够有效 地降低深度学习对大训练数据的依赖.

如图 1(a) 所示, 与通用的图像识别不同, 不同类别的细粒度图像之间仅存在细小而微妙的差异. 细粒度图像的类别通常是同一类物体的不同子类, 例如, 尽管根据图像将狗与袋鼠分类很容易, 但是识 别图 1(a) 中的不同犬种 (金毛寻回犬与拉布拉多犬) 则难度很大, 需要关注细粒度的物体部件 (如金 毛犬的耳朵部分毛较长). 因此, 细粒度的图像识别或分类常需要对图像做详尽的标注, 包括物体及其 各主要部件的位置等, 产生这样的标注信息不但耗时、昂贵, 而且易出错. 细粒度图像的检索如图 1(b) 所示, 检索操作的输入为每行最左边的图像, 其右边 5 张图像是检索的结果, 当返回的结果与输入图 像有相同的细粒度类别时结果正确, 否则即为错误, 图 1(b) 检索返回图像外部有红框的是错误结果. 由于在细粒度图像检索的学习过程中不使用任何标注信息, 其难度较细粒度图像识别更大.

SCDA 方法的核心思想是使用预训练的 VGG16 模型自动发现细粒度图像中物体的相对精确的 位置. 该深度学习模型的高层 (即接近输出层的处理层, 如 Pool5 层) 隐式包含了物体或物体部件的信 息 ${ }^{[13]}$ : 由于 VGG16 是在有 1000 种不同物体的 ILSVRC 数据集上训练的, 我们认为这些信息可以作

\footnotetext{
1) http://www.image-net.org.
} 

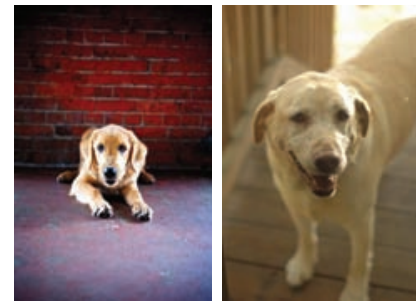

(a)
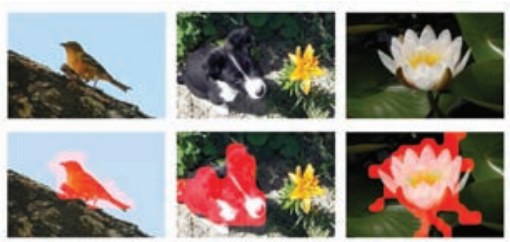

(c)

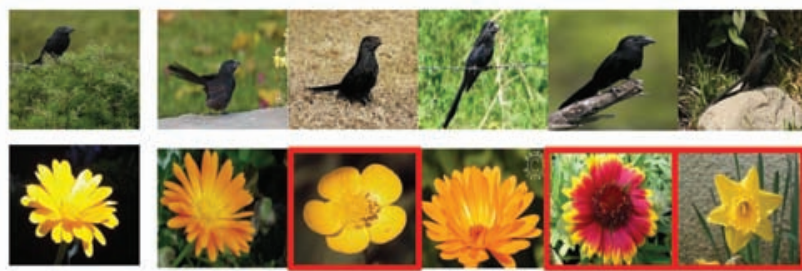

(b)
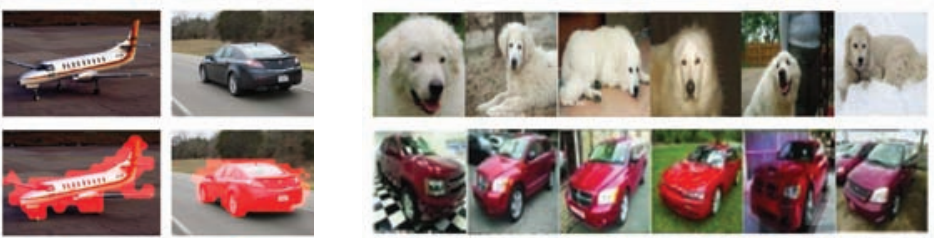

(d)

图 1 (网络版彩图) 细粒度图像检索的 SCDA 方法 ${ }^{[12]}$. (a) 是细粒度图像示例, 左右两张图分别是金毛犬和拉布 拉多犬, (b) 是检索结果样例, 红框表示错误结果, (c) 是定位结果样例, (d) 是 SVD 压缩后的新维度

Figure 1 (Color online) The SCDA method for fine-grained image retrieval [12]. (a) illustrates fine-grained images, with the left and right images being golden retriever and Labrador retriever, respectively; (b) are samples of retrieval results, in which red boxes denote wrong results; (c) shows examples of localization results; and (d) illustrates the new dimensions after SVD

为 “物体” 这个抽象概念的一个有效代表, 并用来将细粒度图像中的物体与背景 (非物体) 区分开来. 具体来说, VGG16 网络的 Pool 5 层产生的激活图是 $7 \times 7 \times 512$ 的张量, 即由 512 个通道 (或神经元) 组 成, 每个通道分别对输入图像中的不同空间位置 (共 $7 \times 7=49$ 个位置) 给出激活强度: 若某通道在某 空间位置激活 (激活强度大于 0 ), 那么该位置对应的图像区域就有可能是若干种物体 (或物体部件) 之 一. 基于这样的解释, SCDA 将每个位置所有通道的激活强度相加, 得到该位置的物体性 (objectness), 若其高于阈值, 认为该位置属于细粒度图像中的物体; 否则的话, 则认为是背景.

给定一张输入图像, 将 VGG16 预训练网络的 Pool5 层激活图记为 $T \in \mathbb{R}^{h \times w \times d}$, 其中 $h=w=7$, $d=512$, 且 $T_{i, j, k}$ 为第 $k$ 个神经元在空间位置 $(i, j)$ 的激活强度, 则如上所述, SCDA 将空间位置 $(i, j)$ 的物体性定义为 $A_{i, j}=\sum_{k=1}^{d} T_{i, j, k}$, 该输入图像的阈值定义为 $\bar{a}=\frac{1}{h w} \sum_{i, j} A_{i, j}$, 则可定义一个掩码矩 阵 $M \in\{0,1\}^{h \times w}$,

$$
M_{i, j}= \begin{cases}1, & \text { if } A_{i, j}>\bar{a}, \\ 0, & \text { otherwise },\end{cases}
$$

1 和 0 分别表示区域 $(i, j)$ 是细粒度图像中的物体区域或背景区域. 进一步假设一张图片中只有一个 主要物体, SCDA 方法仅保留 $M$ 矩阵 (也可以看作是一个 $h \times w$ 大小的二值图像) 中的最大联通区 域, 并将其余较小的掩码为 1 的联通区域改为掩码值 0 . 最后, 使用线性插值方法将 $M$ 放大到与输入 图像相同大小, 即可发现细粒度图像中的物体具体位置. 一些物体位置的检测示例如图 1(c) 所示. 从 图中可以看到, 尽管 SCDA 方法没有直接利用任何训练或标注数据, 仍可以较准确地定位物体. 在此 基础上, SCDA 方法提出使用最大池化 (pooling, 深度网络中的一种常用处理层) 和平均池化的拼接作 为图像的表示, 基于最近邻进行图像检索, 取得了比已有方法更好的检索准确率. 图 1(b) 展示了一些 正确及错误的检索结果, 更多结果请参考文献 [12].

SCDA 方法进一步对这种图像表示通过奇异值分解 (singular value decomposition, SVD) 进行降 维, 得到 256 或者 512 维的新图像表示. 图 1(d) 展示了降维后两个新维度取值最高的图像, 从图中可 

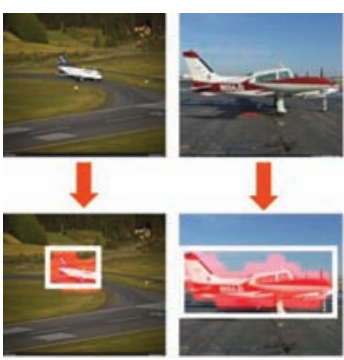
1
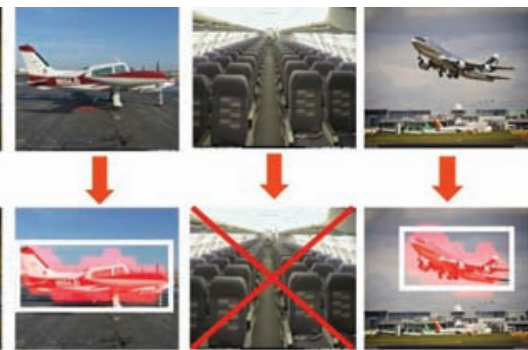

(a)
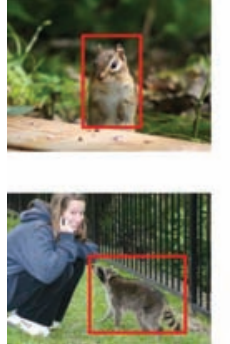
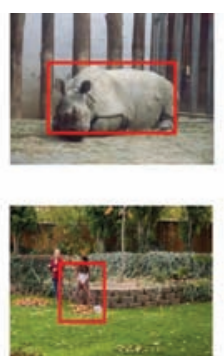

(b)
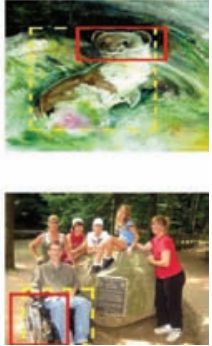

图 2 (网络版彩图) 弱监督的 DDT 方法 ${ }^{[18]}$. (a) 是 DDT 的示意图, 上下两行分别为输入与希望得到的定位结 果, 第 3 张图像中的红色交叉表示该图是噪声, (b) 是 DDT 的定位结果示例, 红色实线框和黄色虚线框分别是真实 的和 DDT 预测的定位结果, 当黄色框不可见时表示我们的定位与真实值完全一致

Figure 2 (Color online) DDT, a weakly supervised method [18]. (a) is an illustration of the DDT task, with the two rows being input images and their respective desired output, and the red cross in the third picture illustrates a noisy input image. (b) contains examples of DDT localization results, with the red solid and yellow dashed boxes being true and DDT predicted localization results, respectively. Our prediction is exactly the same as the groundtruth localization when the yellow box is invisible

以看出, 降维后的图像表示直接对应于细粒度图像中物体的一些微妙属性 (如狗与车的品种、颜色), 这个现象可以为 SCDA 在细粒度图像检索中的成功提供一种合理的解释.

\section{4 降低深度学习对标记的依赖}

事实上, 如果不需要标注信息, 或者需要的标注信息数量较少, 又或者允许标注信息中出现较大 比例的错误以及不确定性, 那么训练样本是相对容易获取的. 就计算机视觉领域而言, 图像搜索引擎 可以提供海量的训练图像, 困难在于针对不同的应用场景为这些图像提供准确而详细的标注. 针对这 个困难, 也有两类有代表性的解决方案: 减少标注的使用数量和自动生成并使用有错误的标记.

随着训练样本数量的增加, 即使其对应的标记包含相当大比例的错误或不确定性 (如 $20 \%$ 甚至 更高), 实验结果表明, 深度学习模型也能从中获益. 因此, 最近一两年来随着搜索引擎技术、大数据 及计算能力的提高, 使用自动生成的, 可能包含很多错误标记的海量样本进行学习表现出令人惊讶的 准确率 ${ }^{[4,14]}$. 这方面的研究尽管在实践中有良好的效果, 但是其理论基础尚待探索, 如包含错误标注 的数据在什么条件下能够提高准确率, 如何在理论指导下更好地利用这样的数据等. 与之相对应的, 另一类方法试图减少对标记信息的使用数量. 这类方法在机器学习领域已经有很多研究, 如半监督学 习 (即使用少部分有标记的数据加上大量无标记的样本进行学习) 或者弱监督学习, 有很多算法与理 论结果 ${ }^{[15]}$. 但是在深度学习领域, 这类方法也处于发展的早期阶段. 半监督方法与深度学习模型的结 合 $[16,17]$ 尽管在一些小型数据集上表现良好, 但是尚有待在复杂大数据上的进一步验证. 在计算机视 觉领域, 弱监督学习则更受欢迎, 即对于每个样本, 减少标记信息的详细程度. 例如, 语义分割的标准 标记需给出图像中每个像素的语义类别, 而在弱监督的深度学习方法中, 只需给出物体的位置框 (4个 坐标即可), 这样大大降低了标注的工作量和难度. 基于这两类方法的思路, 我们分别提出了弱监督的 DDT 方法 [18] 和基于不确定性标注的 DLDL 方法 ${ }^{[19]}$.

DDT 方法用于物体定位与检测, 其使用的监督信息 (标记) 非常少. 给定一个图像数据集, 只需要 约束该数据集中的所有图像都包含同一种共同的 (待定位或检测) 物体即可, 无需给出物体在图像中 的具体位置, 这样的任务被称为物体协同定位或协同检测. 如图 2(a) 所示, DDT 方法的输入为第一行 

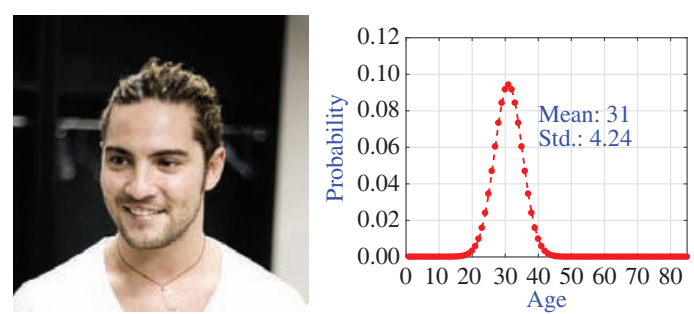

(a)
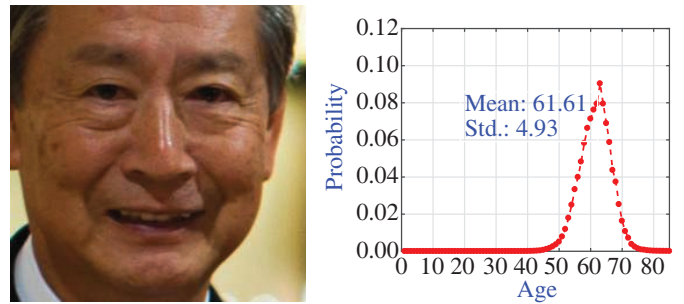

(b)

图 3 (网络版彩图) 利用标记不确定性的 DLDL 方法 ${ }^{[18]}$. (a) 是一张人脸图像及根据多人标注生成的真实标记分 布, (b) 是一张测试图像及 DLDL 方法生成的预测标记分布

Figure 3 (Color online) DLDL, a method that utilizes label uncertainty [18]. (a) is a sample face image and the groundtruth label distribution generated by labels from multiple annotators; (b) is a test image and the prediction by DLDL

的图像 (没有任何标注信息), 包含同一种物体, 其输出为最后一行的位置框. 与多数协同定位、检测方 法不同的是, DDT 允许数据集存在噪声, 如图 2(a) 中的第 3 幅图像并不是飞机.

DDT 的核心思想在于：共同的物体应该具有相同的图像表示, 而背景则应是多种多样的. 与 $\mathrm{SCDA}$ 类似, 预训练的模型将一幅输入图像表示为张量 $T \in \mathbb{R}^{h \times w \times d}$, 即有 $h w$ 个空间区域, 每个区域 表示为 $d$ 维的向量. 若数据集中共有 $n$ 张图像, 那么一共产生 $h w n$ 个表示向量. 在这些向量中, 我们 预计对应于共同物体区域的向量应该互相相似, 而其他向量对应于背景, 则应分布较散乱. 因此, DDT 方法对这 hwn 个向量进行主成分分析操作, 得到均值 $\boldsymbol{\mu}$ 和对应于最大特征值的特征向量 $\boldsymbol{\xi}_{1}, \boldsymbol{\xi}_{1}$ 应该 就是对于共同物体的有效表示. 对于任何一张图像中的一个空间区域, 若其对应的表示向量为 $\boldsymbol{x}$, 那 么判断其是否属于共同物体的成绩为 $p=\boldsymbol{\xi}_{1}^{\mathrm{T}}(\boldsymbol{x}-\boldsymbol{\mu})$. 若 $p>0$, 则认为该区域属于共同物体, 而 $p \leqslant 0$ 则属于背景, 基于这样的成绩计算可以完成物体协同定位. DDT 取得了目前最好的协同定位结果, 一 些结果如图 2(b) 所示, 使用的预训练模型为 VGG19. VGG19 是基于 ILSVRC 训练的, 值得注意的是, 图 2(b) 的 6 个类别的物体并没有出现在 ILSVRC 中, 因此 DDT 方法具有很好的可推广性.

我们提出的另外一种方法 DLDL 有效利用不确定性的标记来同时降低对数据和标记的依赖. DLDL 将标记分布学习 (label distribution learning) ${ }^{[20]}$ 的思想与深度学习成功地进行了结合. 尽管 机器学习在多数情况下假设训练集中样本的标记是准确且确定的, 现实世界的数据很多时候并不符合 这个假设. 图 3(a) 来自 ChaLearn LaP 系列竞赛 2 , 展示了人脸年龄估计的不确定性, 不同的标注人员 会基于图 3(a) 中左边那副图给出不同的年龄估计数值, 而且不同人标注的年龄可能会相差很大. LaP 竞赛收集数据时, 计算多人标记结果的均值和标准差, 将这两个数值作为标记; 换句话说, 该标记不是 单个年龄值, 而是一个年龄的概率分布 (distribution), 其均值和标准差见图 3(a) 中右边那副图. 针对 这种标记的不确定性, Geng 等 [20] 提出了标记分布学习 LDL, 即学习算法的输出同样为一个分布, 通 过使得学习算法的输出分布与标记的真实分布距离最小来训练模型的参数. Geng 等将 LDL 成功地 应用于人脸年龄估计、头部姿态估计等应用场景, 成为了这些领域的通行方法.

然而, LDL 方法有一些缺陷难以处理, 例如其只能使用手工设计的视觉特征, 从而无法利用深 度学习在特征表示方面端到端学习的优势. 为了克服这些困难, 我们提出了深度标记分布学习方法 DLDL ${ }^{[19]}$, 这是一种端到端 (即深度网络的输入是原始输入而输出直接是需要预测的量) 的深度学习 方法. 由于深度网络的输出可以为向量或矩阵, 我们将标记的分布 (可以是一维, 二维, 甚至更高维的 分布) 直接作为深度网络的输出, 并定义相对熵 (relative entropy) 作为两个分布相似度的度量. 这样,

2) http://chalearnlap.cvc.uab.es/challenge/12/description/. 
只需要生成合适的真实标记分布, 就可以通过随机梯度下降算法进行深度标记分布学习, 整个方法的 框架与传统的深度学习差异很小. 若具体的应用场景需要单个数值作为输出 (例如在基于人脸图像的 年龄估计中, 预测结果最好是一个数字而不是一个分布), 在得到网络的预测分布之后, 可以使用分布 的期望. 图 3(b) 展示了一幅用于测试的图像 (左图, 来自 LaP 竞赛) 以及 DLDL 给出的预测结果 (右 图), 该图人工标注的标记分布均值为 62 岁,而 DLDL 预测的分布均值为 61.61 岁. DLDL 是一个端到 端的网络学习方法, 因此可以自主学习好的图像表示, 并且可以很方便地处理非参数化的分布, 取得 了比 LDL 更好的预测准确度. 我们还将 DLDL 推广到多标记图像识别及图像语义分割, 详情请参考 文献 [19].

更重要的是, DLDL 从两个角度减轻了对于训练数据和标记的需求, 首先, 由于通过大数据在相似 领域学习得到的深度图像表示具有很好的通用性, 可以用类似领域预训练的网络初始化并进行微调. 在 $\mathrm{LaP}$ 竞赛的数据上, 使用人脸识别的网络 $\mathrm{VGG}-\mathrm{Face}^{3)}$ 进行微调, 即使 $\mathrm{LaP}$ 竞赛的训练数据很少 (少于 2500 张), 也可以得到很准确的年龄预测. 其次, DLDL 隐式利用了标记的不确定性, 例如, 若某 人脸图像被标记为 25 岁, 则该人年龄为相邻年龄 (如 24 或 26 岁) 的可能性也很大, 可以作为相邻年 龄的训练数据, DLDL 自动利用了这种关联特性, 但不需要显式生成更多数据.

\section{5 压缩深度模型的大小和计算量}

压缩深度学习模型对存储与运算量等资源的需求是深度学习目前的研究热点. 值得注意的是, 模 型大小、运行时间、电力占用等因素在很多时候是互相关联的, 一个设计良好的模型如果有较少的参 数, 那么它的运算量也可能会相对较少, 并通常意味着消耗较少的电量. 因此, 本节综合考虑这些方面 的压缩方法.

对深度网络各方面资源占用的压缩方法多种多样. 一种思路是将网络中的参数从浮点数 (一般占 用 32 个比特) 改为二值 (取值为 0 或 1 , 占用 1 个比特), 有些方法进一步将网络各层产生的激活图也 变为二值. 由于二值变量的运算远快于浮点运算, 这个系列的方法不但能够将网络大小压缩 32 倍, 还 能大大加速网络的运算速度 ${ }^{[21]}$. 然而, 由浮点变为二值变量会带来网络预测准确率的很大降低. 另一 种常见思路是通过近似算法来加速网络的运算, 例如将浮点数量化为 $2 \sim 4$ 个比特, 或者将向量量化进 行近似计算 ${ }^{[22]}$. 这种思路一般能取得近似于原网络的准确率, 但是由于近似计算需要特别的软件包 或硬件支持, 通用性受到一定限制, 如在 TensorFlow 深度学习框架中提供了将浮点数量化为 8 位定点 数的功能以促进深度网络在移动平台的部署. 第 3 种常见思路是基于稀疏 (sparse) 学习将网络的部分 参数或参数组在学习过程中置为 0 , 这样可以省去部分运算量以及减少网络需要保存的参数个数 ${ }^{[23]}$. 然而, 由于目前的软硬件系统对稀疏矩阵的运算支持不足, 这种方法在实际应用中的效果尚有待提高. 目前比较常见的另一种思路是设计新的深度网络结构, 在不降低准确率的前提下降低计算量和参数个 数, 如 ResNeXt ${ }^{[24]}$, SqueezeNet ${ }^{4)}$, MobileNet $^{5)}$, ShuffleNet ${ }^{6)}$ 等. 这些新型网络结构通常需要特别的软 硬件支持, 才能完全发挥其优势.

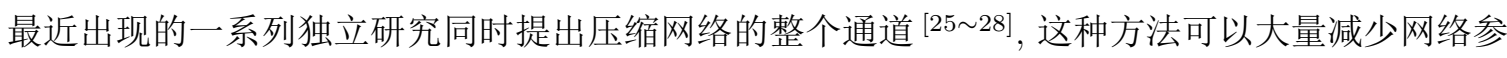
数 (即模型大小) 但保持与原网络相似的准确率, 减少网络中激活图的大小 (从而有利于在移动端的

\footnotetext{
3) http://www.robots.ox.ac.uk/ vgg/software/vgg_face/.

4) SqueezeNet. https://arxiv.org/abs/1602.07360.

5) MobileNets. https://arxiv.org/abs/1704.04861.

6) ShuffleNet. https://arxiv.org/abs/1707.01083.
} 


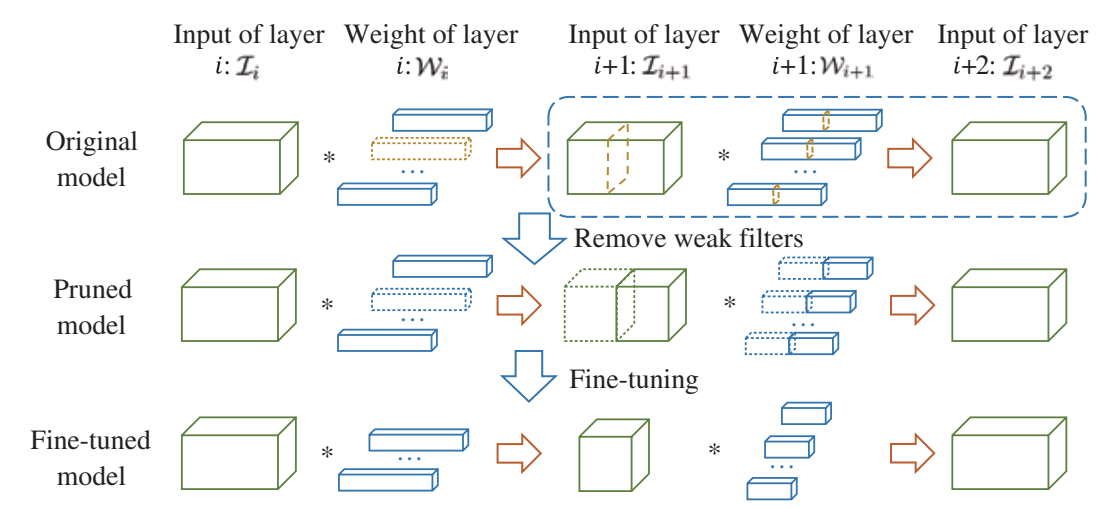

图 4 (网络版彩图) ThiNet 方法的主要思路 ${ }^{[25]}$ 是使用下一层的输出来指导网络当前层的压缩, 并随后进行微调

Figure 4 (Color online) Ideas behind the ThiNet method [25]: using the next layer's output to guide the compression of the current layer, and then fine-tune the network

部署)、同时可以极大地减少网络的运算量. 压缩整个通道的另一个优点是网络运算所需要的软硬件 支持与原网络没有任何变化, 因此适用于任何软硬件环境, 具有很强的通用性. 我们提出的 ThiNet 方 法 [25] 就是压缩整个通道, 但是与其他方法不同的是, 我们的理论推导表明应该用多个网络层协同压 缩当前层才是最优的, 而其他方法则只采用当前层的信息.

如图 4 所示, 若当前层为第 $i$ 层, 我们的目标是去除其参数 $\mathcal{W}_{i}$ 中的若干通道, 但不影响整个网络 的运算. 若 $\mathcal{W}_{i}$ 中的某通道被去除, 则第 $i+1$ 层的输入 $\mathcal{I}_{i+1}$ 与参数 $\mathcal{W}_{i+1}$ 中相应的通道也需要被移 除. 然而, 由于 $\mathcal{W}_{i+1}$ 的卷积核个数没有变化, 第 $i+1$ 层的输出 (同时也是第 $i+2$ 层的输入 $\mathcal{I}_{i+2}$ ) 维 度保持不变. 因此, 我们移除当前层 (第 $i$ 层) 中的通道需要遵循的准则是: 使得第 $i+1$ 层的输出在移 除前后尽量保持不变, 即, 需要用下一层的输出, 而不是当前层的输出来指导当前层的压缩, 这是我们 的 ThiNet 方法与其他方法主要的不同之处.

ThiNet 方法基于以上的理论分析设计, 并取得了良好的效果. 以 VGG16 网络为例, ThiNet 在移 除各卷积层一半通道的情况下, 可以将网络的参数大大减少, 浮点运算数量减少到原网络的三分之一 以下, 同时在 ILSVRC 上获得比原网络还要高的准确率. 同时, 我们将压缩后的网络推广到与 ILSVRC 不同的问题领域, 也取得了良好的效果.

\section{6 讨论与结语}

本文介绍了资源受限的深度学习这一新问题和其中蕴含的挑战, 简要介绍了这一领域内的一些研 究进展, 并以我们在计算机视觉领域的研究实践为例, 分别对压缩深度学习模型对数据、标记、计算这 3 种重要资源需求的方法进行介绍.

资源受限的深度学习不但有很好的研究价值, 而且对于深度学习直接应用到我们日常生活的方方 面面至关重要. 这一领域还有很多公开问题没有被解决, 值得进行深入的研究和应用. 从我们的研究 实践来看, 以下 3 个方面的研究值得进一步关注:

(1) 针对具体的应用环境, 软硬件结合设计新的软件框架与硬件系统, 在移动端部署深度网络.

(2) 将半监督学习与深度网络有机结合, 研究新的深度半监督学习方法以充分利用无标记的大 数据.

(3) 充分理解深度网络各层的运行机制, 研究新的网络层与网络结构, 以降低参数冗余与计算量. 


\section{参考文献}

1 Krizhenvsky A, Sutskever I, Hinton G. ImageNet classificaiton with deep convolutional neural networks. In: Proceedings of the 25th Advances in Neural Information Processing Systems, Lake Tahoe, 2012. 1097-1105

2 LeCun Y, Bottou L, Bengio Y, et al. Gradient-based learning applied to document recognition. Proc IEEE, 1998, 86: $2278-2324$

3 He K M, Zhang X Y, Ren S Q, et al. Deep residual learning for image recognition. In: Proceedings of the IEEE International Conference on Computer Vision and Pattern Recognition, Las Vegas, 2016. 770-778

4 Sun C, Shrivastava A, Singh S, et al. Revisiting unreasonable effectiveness of data in deep learning era. In: Proceedings of the International Conference on Computer Vision, Venice, 2017. 843-852

5 Simonyan K, Zisserman A. Very deep convolutional networks for large-scale image recognition. ArXiv: 1409.1556

6 Kokkinos I. UberNet: training a universal convolutional neural network for low-, mid-, and high-level vision using diverse datasets and limited memory. In: Proceedings of the IEEE International Conference on Computer Vision and Pattern Recognition, Hawaii, 2017. 6129-6138

7 Goodfellow I, Pouget-Abadie J, Mirza M, et al. Generative adversarial nets. In: Proceedings of the 27th Advances in Neural Information Processing Systems, Montreal, 2014. 2672-2680

8 Shrivastava A, Pfister T, Tuzel O, et al. Learning from simulated and unsupervised images through adversarial training. In: Proceedings of the IEEE International Conference on Computer Vision and Pattern Recognition, Hawaii, 2017. $2107-2116$

9 Russakovsky O, Deng J, Su H, et al. ImageNet large scale visual recognition challenge. Int J Comput Vision, 2015, 115: $211-252$

10 Ren S Q, He K M, Girshick R, et al. Faster R-CNN: towards real-time object detection with region proposal networks. In: Proceedings of the 28th Advances in Neural Information Processing Systems, Montreal, 2015. 91-99

11 Shelhamer E, Long J, Darrell T. Fully convolutional networks for semantic segmentation. IEEE Trans Pattern Anal Mach Intell, 2017, 39: 640-651

12 Wei X-S, Luo J-H, Wu J X, et al. Selective convolutional descriptor aggregation for fine-grained image retrieval. IEEE Trans Image Process, 2017, 26: 2868-2881

13 Zeiler M D, Fergus R. Visualizing and understanding convolutional networks. In: Proceedings of the European Conference on Computer Vision, Zurich, 2014. 8689: 818-833

14 Krause J, Sapp B, Howard A, et al. The unreasonable effectivenss of noisy data for fine-grained recognition. In: Proceedings of the European Conference on Computer Vision, Amsterdam, 2016. 9907: 301-320

15 Zhu X, Goldberg A B. Introduction to Semi-Supervised Learning. San Rafael: Morgan \& Claypool Publishers LLC, 2009

16 Rasmus A, Valpola H, Honkala M, et al. Semi-supervised learning with ladder networks. In: Proceedings of the Advances in Neural Information Processing Systems 28, Montreal, 2015. 3546-3554

17 Laine S, Aila T. Temporal ensembling for semi-supervised leanring. In: Proceedings of the International Conference on Learning Representations, Toulon, 2017

18 Wei X-S, Zhang C-L, Li Y, et al. Deep descriptor transforming for image co-localization. In: Proceedings of the 26th International Joint Conference on Artificial Intelligence, Melbourne, 2017. 3048-3054

19 Gao B-B, Xing C, Xie C-W, et al. Deep label distribution learning with label ambiguity. IEEE Trans Image Process, 2017, 26: $2825-2838$

20 Geng X, Ji R Z. Label distribution learning. IEEE Trans Data Eng, 2016, 28: 1734-1748

21 Rastegari M, Ordonez V, Redmon J, et al. XNOR-Net: imageNet classification using binary convolutional neural networks. In: Proceedings of the European Conference on Computer Vision, Amsterdam, 2016, 9908: 525-542

22 Wu J X, Leng C, Wang Y H, et al. Quantized convolutional neural networks for mobile devices. In: Proceedings of the IEEE International Conference on Computer Vision and Pattern Recognition, Las Vegas, 2016. 4820-4828

23 Han S, Pool J, Tran J, et al. Learning both weights and connections for efficient neural network. In: Proceedigns of the Advances in Neural Information Processing Systems 28, Montreal, 2015. 1135-1143

24 Xie S, Girshick R, Dollar P, et al. Aggregated residual transformations for deep neural networks. In: Proceedings of the IEEE International Conference on Computer Vision and Pattern Recognition, Hawaii, 2017. 1492-1500

25 Luo J-H, Wu J X, Lin W. ThiNet: a filter level pruning method for deep neural network compression. In: Proceedings 
of the International Conference on Computer Vision, Venice, 2017. 5058-5066

26 He Y H, Zhang X Y, Sun J. Channel pruning for accelerating very deep neural networks. In: Proceedings of the International Conference on Computer Vision, Venice, 2017. 1389-1397

27 Liu Z, Li J G, Shen Z Q, et al. Learning efficient convolutional networks through network slimming. In: Proceedings of the International Conference on Computer Vision, Venice, 2017. 2736-2744

$28 \mathrm{Li} \mathrm{H}$, Kadav A, Durdanovic I, et al. Pruning filters for efficient ConvNets. In: Proceedings of the International Conference on Learning Representations, Toulon, 2017

\title{
Resource-constrained deep learning: challenges and practices
}

\author{
Jianxin WU*, Bin-Bin GAO, Xiu-Shen WEI \& Jian-Hao LUO \\ National Key Laboratory for Novel Software Technology, Nanjing University, Nanjing 210023, China \\ * Corresponding author. E-mail: wujx2001@nju.edu.cn
}

\begin{abstract}
Deep learning has made significant progress in recent years. However, deep learning models require many computation-related resources, and their learning process requires a large number of data points and their labels. Hence, the reduction of resource consumption of deep learning, i.e., resource-constrained deep learning, is a current research focus. In this study, we first analyze deep learning's thirst for various types of resources and the challenges they lead to and thereafter briefly introduce research progress from three aspects: data, label, and computation resources. Further, we provide detailed introductions of these areas using our research results as examples.
\end{abstract}

Keywords deep learning, resource constraints, data resources, label resources, computation resources

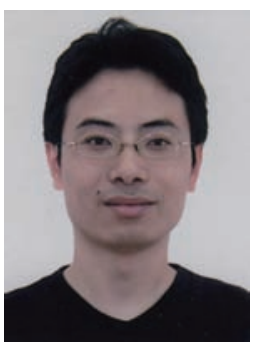

Jianxin WU received his B.S. and M.S. degrees from Nanjing University, Nanjing, in 1999 and 2002, respectively, and his Ph.D. degree in computer science from Georgia Institute of Technology, Atlanta, USA in 2002. He is currently a professor in the Department of Computer Science and Technology, Nanjing University. His current research interests include computer vision and machine learning.

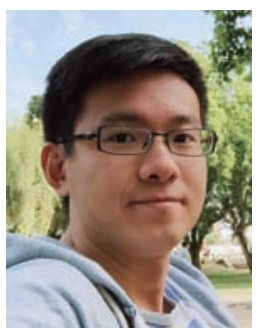

Xiu-Shen WEI received his B.S. degree in computer science and technology in 2012. He is currently a Ph.D. candidate in the Department of Computer Science and Technology, Nanjing University, China. His research interests include computer vision and machine learning.

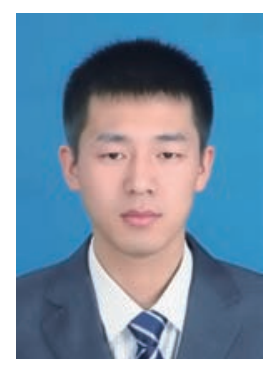

Bin-Bin GAO received his B.S. and M.S. degrees in applied mathematics in 2010 and 2013, respectively. He is currently pursuing his Ph.D. degree in the Department of Computer Science and Technology, Nanjing University, China. His research interests include computer vision and machine learning.

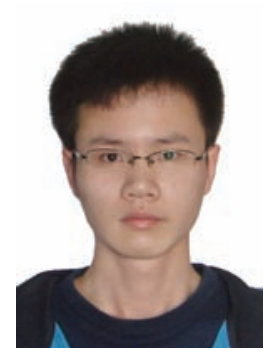

Jian-Hao LUO received his B.S. degree from College of Computer Science and Technology, Jilin University, China in 2015. He is currently working toward his Ph.D. degree in the Department of Computer Science and Technology, Nanjing University, China. His research interests include computer vision and machine learning. 